\title{
Deep Inferior Epigastric Perforator Flap Breast Reconstruction in a Patient with Huge Uterine Leiomyoma
}

\section{Ji Min Kim, Jun Gul Ko, Deuk Young Oh}

Department of Plastic and Reconstructive Surgery, Seoul St. Mary's Hospital, College of Medicine, The Catholic University of Korea, Seoul, Korea

No potential conflict of interest relevant to this article was reported.
The deep inferior epigastric perforator (DIEP) flap has long been the gold standard for autologous breast reconstruction. Various factors that can disrupt abdominal wall vascular anatomy make dissection of the DIEP flap a technically demanding procedure. The mass effect from a huge uterine leiomyoma has not been previously reported as a factor influencing abdominal wall vascularity. The aim of this report was to describe the case of a patient with a huge uterine leiomyoma who underwent immediate free DIEP flap breast reconstruction without any major complications.

Keywords Free tissue flaps, Leiomyoma, Mammaplasty, Microsurgery, Perforator flap

\section{INTRODUCTION}

The deep inferior epigastric perforator (DIEP) flap has long been considered the standard procedure for autologous breast reconstruction. While the anatomy of the deep inferior epigastric artery (DIEA) has been reported to vary among individuals, the DIEP flap procedure has been shown to be reliable [1,2]. Various factors that can disrupt abdominal wall vascular anatomy make dissection of the DIEP flap a technically demanding procedure. For this reason, preoperative mapping of the perforator using multi-detector computed tomography (MDCT) has been proven to be cost-effective $[3,4]$.

The mass effect from a huge uterine leiomyoma has not been reported as a factor impacting abdominal wall vascularity. In this study, the authors successfully performed DIEP flap breast reconstruction in a patient who had a $19 \times 19 \mathrm{~cm}$ uterine leiomyoma and a distended abdomen.

Received: Mar 6, 2017 Revised: Apr 11, 2017 Accepted: Apr 12, 2017 Correspondence: Deuk Young Oh Department of Plastic and Reconstructive Surgery, Seoul St. Mary's Hospital, College of Medicine, The Catholic University of Korea, 222 Banpo-daero, Seocho-gu, Seoul 06591, Korea. E-mail: ohdeuk1234@hanmail.net

Copyright () 2017 The Korean Society for Aesthetic Plastic Surgery.

This is an Open Access article distributed under the terms of the Creative Commons Attribution Non-Commercial License (http://creativecommons.org/licenses/by-nc/4.0/) which permits unrestricted non-commercial use, distribution, and reproduction in any medium, provided the original work is properly cited. www.e-aaps.org

\section{CASE}

A 44-year-old patient was diagnosed with left-sided breast cancer, and she requested immediate breast reconstruction. In the radiology report, an irregular, speculated, enhancing mass measuring 2.4 $\times 2.2 \times 2.6 \mathrm{~cm}$ was found, corresponding to the 4 o'clock position on the left side of the breast, located $3.3 \mathrm{~cm}$ away from the nipple. Microcalcifications and ductal extension were also found. In addition, a $2.2 \mathrm{~cm}$ enlarged lymph node was identified in level I of the left axilla. The mass was confirmed as invasive ductal carcinoma by histologic examination through a core-needle biopsy. Additionally, a $1 \times 1.1 \mathrm{~cm}$ rim-enhancing nodule was identified at the 12 oclock position on the right breast, and results of the core-needle biopsy indicated nodular adenosis.

The patient was a nurse, unmarried, not pregnant, and had learned of her uterine leiomyoma about 4 years to 5 years ago. At that time, she did not undergo surgery for the leiomyoma for personal reasons, but wanted surgical treatment for the leiomyoma at the same time as her breast reconstruction.

MDCT was performed to analyze abdominal wall vascularity, and the scan confirmed a $17 \mathrm{~cm}$ huge uterine leiomyoma with high vascularity (Fig. 1 and 2)

Due to at least 4 years of mass effect from the leiomyoma, the bilateral deep inferior epigastric vessels had become relatively thin, and we suspected that compression of the vascular pedicle may have occurred. Consequently, we planned to use the superficial inferior epigastric vein (SIEV) if the deep inferior epigastric vein (DIEV) 


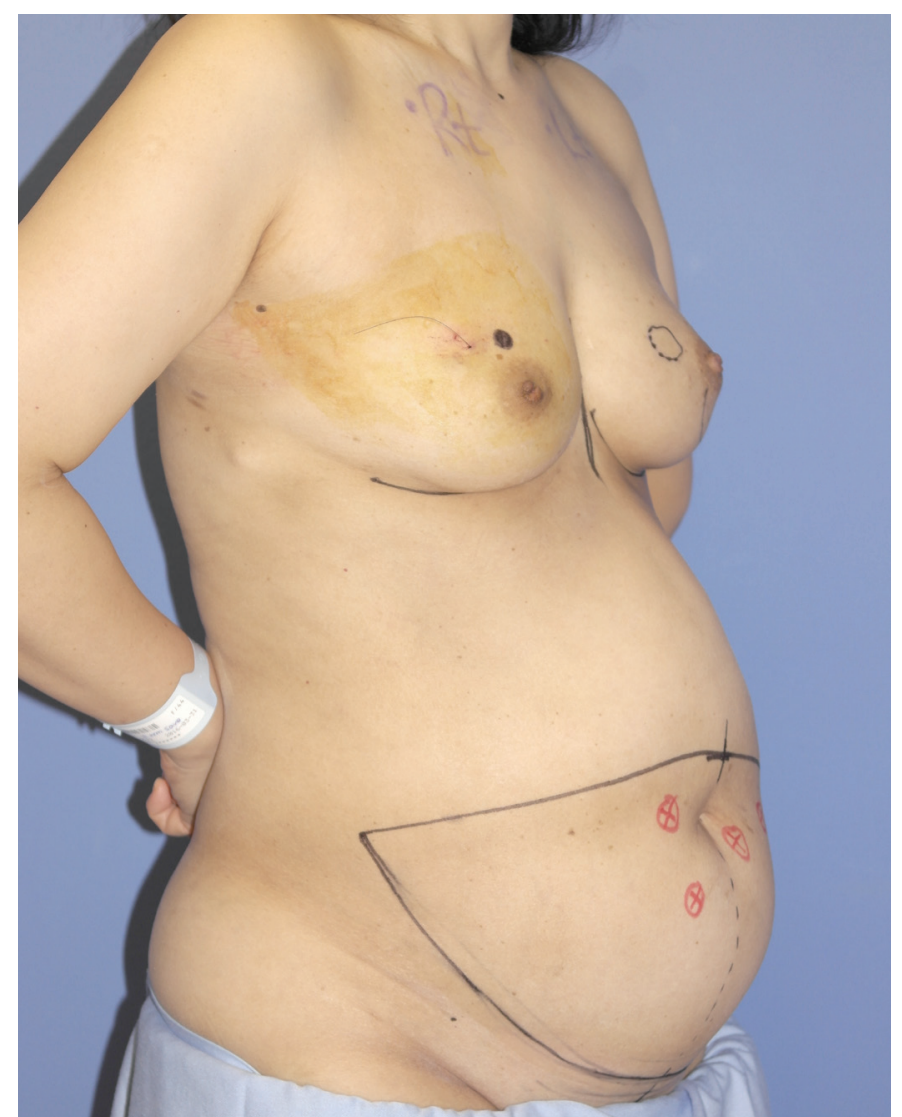

Fig. 1. Preoperative photograph showing the patient's protruding lower abdomen.

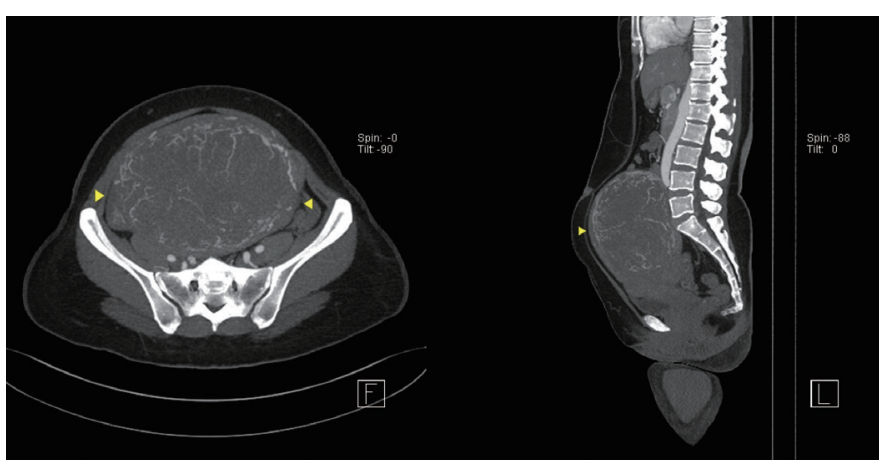

Fig. 2. Preoperative computed tomography angiography findings. A $17 \mathrm{~cm}$ huge uterine leiomyoma with multiple smaller uterine leiomyomas was found in the pelvic cavity (yellow arrowheads).

was unreliable. After a nipple-sparing mastectomy and a sentinel lymph node biopsy, immediate breast reconstruction using a DIEP flap and a total hysterectomy were planned for the patient in cooperation with gynecologic surgeons.

Breast surgeons and plastic surgeons performed the operation using a two-team approach. The mass in the right breast was excised and biopsied, with a final pathology report indicating adeno-

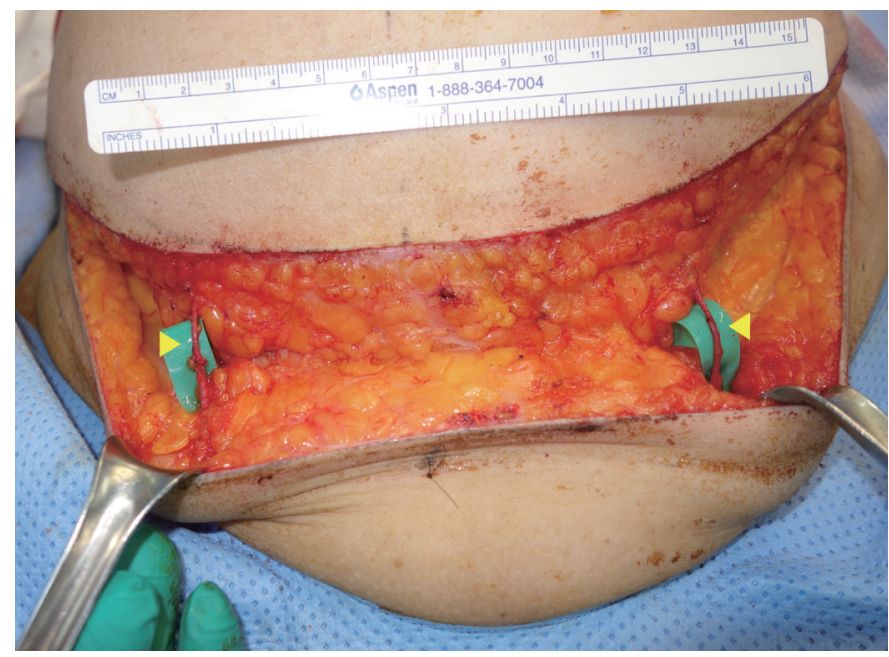

Fig. 3. Intraoperative findings. The diameter of the left superficial inferior epigastric vein (SIEV) was $2.1 \mathrm{~mm}$, and the diameter of the right SIEV was $1.7 \mathrm{~mm}$ (yellow arrowheads).

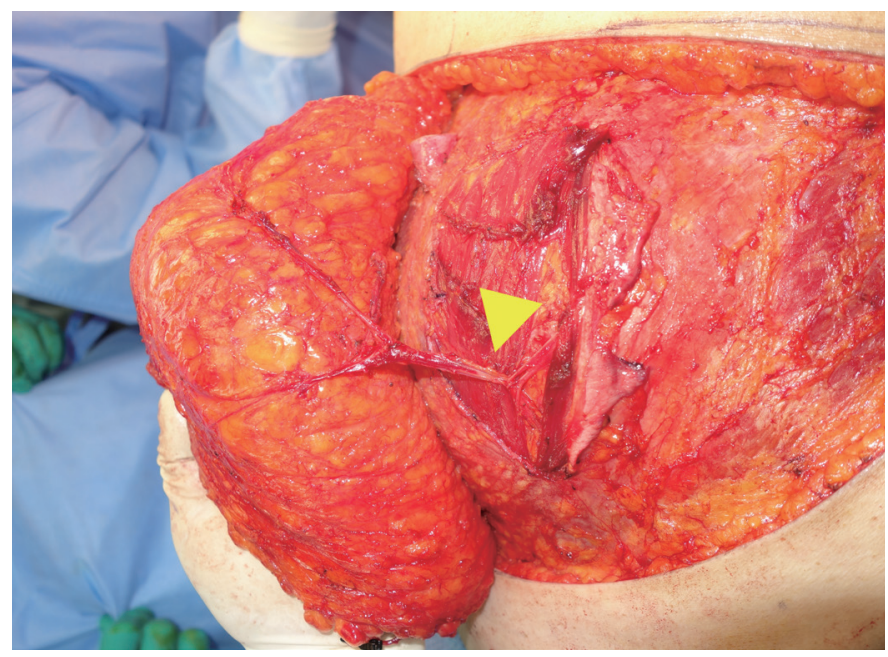

Fig. 4. Intraoperative findings. The image shown was taken after the dissection and identification of the most prominent deep inferior epigastric perforator vessels on the left side (yellow arrowhead). They were average in size.

myoepithelioma. Nipple-sparing mastectomy and axillary sentinel lymph node biopsy were performed on the left breast. Results of the frozen biopsy showed no metastasis of the sentinel lymph node, so axillary lymph node dissection was not performed.

The plastic surgery team simultaneously performed flap elevation, and the SIEV was identified during elevation of the DIEP flap. The diameters of the left SIEV and right SIEV were $2.1 \mathrm{~mm}$ and 1.7 $\mathrm{mm}$, respectively (Fig. 3), and both were within the average size ranges [5]. The most prominent DIEP vessels on the left side were identified (Fig. 4). The diameters of the DIEA and DIEV were 2.1 $\mathrm{mm}$ and $2.5 \mathrm{~mm}$, respectively, which were within the average size 


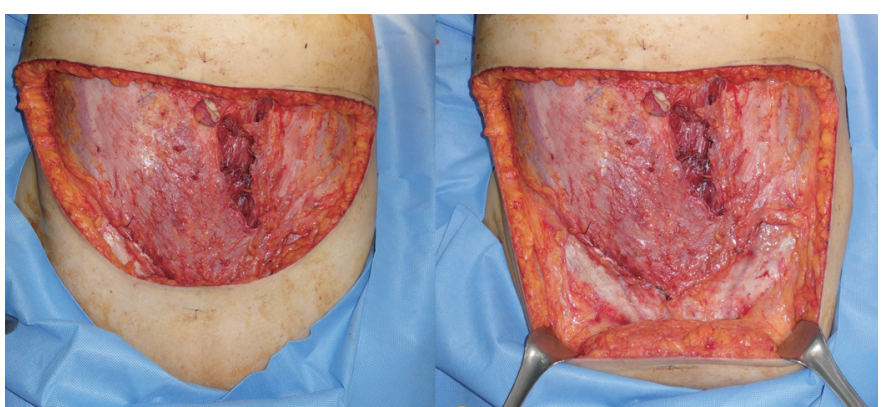

Fig. 5. The image shown was taken after total abdominal hysterectomy via a Pfannenstiel incision on the peritoneum.

ranges. After the right DIEP flap and both SIEVs were clamped to prevent blood flow, the condition of the flap was examined. The color, refill, and turgor of a part of zones I, III, and II were verified to be intact, and we confirmed that only the left DIEA and left DIEV were sufficient for whole flap survival. Therefore, we decided to perform anastomosis with only the left DIEP flap. After identifying the left thoracodorsal artery and vein, the DIEP flap was divided and elevated. The weight of the harvested flap was $1,141 \mathrm{~g}$. According to our protocol, the harvested flap was then infused with iced normal saline and inverted every 15 minutes. Afterward, the gynecology team performed a total abdominal hysterectomy using an isolated Pfannenstiel incision (Fig. 5), which took approximately 50 minutes. Finally, the plastic surgery team anastomosed the left DIEA and left DIEV to the thoracodorsal artery and vein and performed donor site closure without complications (Fig. 6). The weight of the insetting flap was $539 \mathrm{~g}$. The operation ended without any difficulty, and the patient was discharged on postoperative day 8.

The patient has been under follow-up observation with no major breast or abdominal wall complications to date.

\section{DISCUSSION}

Since the introduction of the DIEP flap, immediate or delayed breast reconstruction has frequently been performed following mastectomy. However, immediate DIEP flap breast reconstruction in a patient with a huge uterine leiomyoma has not been previously reported. This may be due to the vascular anatomy of the perforator flap. As the vascular patency of the abdominal wall becomes unclear from the mass effect of a massive leiomyoma, surgeons hesitate to consider the DIEP flap as the treatment of choice under such circumstances.

One challenge to DIEP flap breast reconstruction is venous congestion. To cope with this complication, superdrainage using the superficial epigastric vein has been proven effective [6-11]. We estimated the diameter of the vessel through MDCT preoperatively, and we preserved every perforator and pedicle, including all DIEAs, DIEVs, SIEVs, and superficial inferior epigastric arteries during

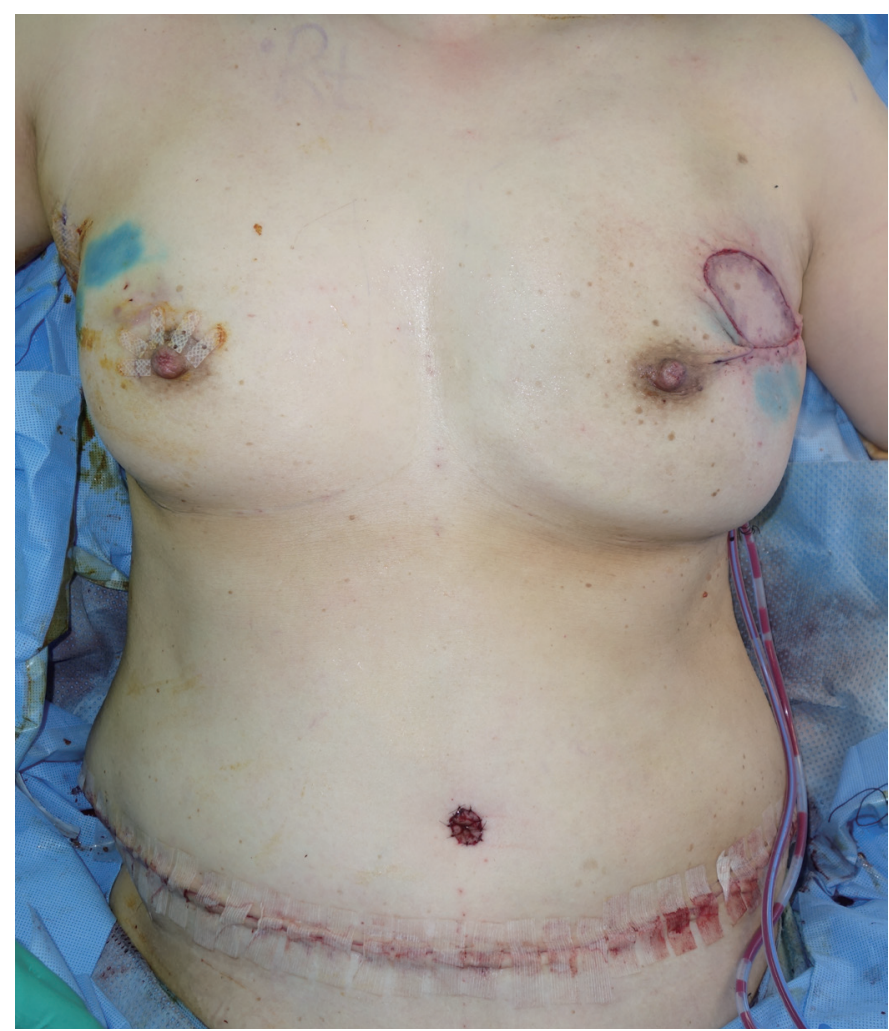

Fig. 6. Immediate postoperative photograph.

dissection of the flap. Additionally, we were able to predict the viability of the flap through vessel clamping during flap elevation. Using this procedure, the authors confirmed the left DIEA and left DIEV to be sufficient for whole flap survival. This procedure can minimize unnecessary vascular anastomoses, lower a surgeon's stress, and decrease surgery time.

During a normal pregnancy, the growing uterus also causes a mass effect on the abdominal wall. In the second trimester, the size of the uterus begins to increase from the inside of the pelvis, and in the 20th week, the fundal height (i.e., the distance in centimeters between the uterine fundus and the pubic bone) normally coincides with the number of weeks pregnant. In the third trimester, the size of the uterus generally increases from the pubic area to the bottom of the rib cage and moves upward. Therefore, the period during which the mass effect actually occurs during a normal pregnancy is thought to be roughly less than 20 weeks, and then the uterus gradually returns to its pre-pregnancy size and position over the span of approximately 6 weeks after the involution phase.

In the patient described in this study, a persistent mass effect occurred for at least 4 years. Furthermore, the characteristics and location of the leiomyoma did not significantly change over time, unlike the changes that occur during a normal pregnancy. Additionally, the mass effect occurred gradually in the lower abdomen, which is where the vessel that is most commonly used for the DIEP flap is located. For all of these reasons, this case could not be used 
to study the mass effect and its impact on vessel size that occurs when the uterus expands during pregnancy, and we believe that additional research is needed on this issue.

The mass effect may either reduce vascularity of the main vessel through its compression or increase vascularity through expansion of the collateral vessel. We will continue to identify patients similar to the patient described in this case study and conduct additional research if possible.

In this study, the ischemic and cold states of the harvested flap were prolonged because of the additional operation time required to perform the hysterectomy (approximately 50 minutes). In general, when performing autologous breast reconstruction with the DIEP flap, reperfusion to the flap is usually detected immediately after vascular anastomosis by Doppler sonography or after bleeding at the flap margin. We confirmed that reperfusion occurred approximately 10 minutes after anastomosis, and this was probably because of the 50-minute surgical delay.

In accordance with our surgical protocol, the harvested flap was infused with iced normal saline and inverted every 15 minutes. Animal and experimental studies have shown that elevated flaps can be kept in cold ischemia to provide a longer, yet safe, time to use the free flaps [12-15]. In addition, it is common to use hypothermia to prolong the amount of tolerated ischemia time in solid organ transplantation, such as the liver and kidney. The effect of warm ischemia on living tissues is well known, so immediate anastomosis should be performed during free flap breast reconstruction. Indeed, many surgeons perform rapid anastomosis before an ischemic complication can occur. However, elevating the DIEP flap during flap harvesting may be very mentally and physically tiring for a surgeon, and a long operation without rest may adversely affect the surgical outcome. Therefore, leaving flaps in cold ischemia can provide extra time. Lee and Lee [12] also reported that cold ischemia time up to 5 hours is safe, and is not associated with an increased risk of flap complications. Consequently, we cooled the flap, allowing us to prolong the ischemia time without compromising the success of the operation.

This case was the first of its kind. Going forward, we believe that it may be best to consult gynecologic surgeons regarding pedicle division following hysterectomy if similar cases are found.

\section{CONCLUSIONS}

With a thorough grounding in surgical principles, we were able to perform a DIEP flap breast reconstruction in the unique circumstances presented by this case. Of particular note, preoperative MD$\mathrm{CT}$ and intraoperative perforator vessel clamping were found to be critically important.

Ultimately, cooling of the flap and prolonging ischemia time provided the gynecology team with additional time to successfully perform a total abdominal hysterectomy without any flap complications.

\section{PATIENT CONSENT}

Patients provided written consent for the use of their images.

\section{REFERENCES}

1. Allen RJ, Treece P. Deep inferior epigastric perforator flap for breast reconstruction. Ann Plast Surg 1994;32:32-8.

2. Blondeel PN. One hundred free DIEP flap breast reconstructions: a personal experience. Br J Plast Surg 1999;52:104-11.

3. Hughes JM, Smith JR, Jones L, et al. Incidental findings in CT angiograms for free DIEP flap breast reconstruction - Do they change our management? Eur J Surg Oncol 2016;42:59-63.

4. Minqiang X, Lanhua M, Jie L, et al. The value of multidetector-row CT angiography for pre-operative planning of breast reconstruction with deep inferior epigastric arterial perforator flaps. Br J Radiol 2010; 83:40-3.

5. Ayhan S, Oktar SO, Tuncer S, et al. Correlation between vessel diameters of superficial and deep inferior epigastric systems: Doppler ultrasound assessment. J Plast Reconstr Aesthet Surg 2009;62:1140-7.

6. Villafane O, Gahankari D, Webster M. Superficial inferior epigastric vein (SIEV): 'lifeboat' for DIEP/TRAM flaps. Br J Plast Surg 1999;52:599.

7. Sojitra NM, Vandevoort M, Ghali S, et al. Two new techniques for correcting venous congestion in the free DIEP flap for breast reconstruction: an analysis of venous augmentation in 581 DIEP flaps. Plast Reconstr Surg 2010;125:72e-4e.

8. Tutor EG, Auba C, Benito A, et al. Easy venous superdrainage in DIEP flap breast reconstruction through the intercostal branch. J Reconstr Microsurg 2002;18:595-8.

9. Blondeel PN, Arnstein M, Verstraete K, et al. Venous congestion and blood flow in free transverse rectus abdominis myocutaneous and deep inferior epigastric perforator flaps. Plast Reconstr Surg 2000;106:1295-9.

10. Schaverien MV, Ludman CN, Neil-Dwyer J, et al. Relationship between venous congestion and intraflap venous anatomy in DIEP flaps using contrast-enhanced magnetic resonance angiography. Plast Reconstr Surg 2010;126:385-92.

11. Lee KT, Mun GH. Benefits of superdrainage using SIEV in DIEP flap breast reconstruction: A systematic review and meta-analysis. Microsurgery 2017;37:75-83.

12. Lee DT, Lee G. Cold ischemia in microvascular breast reconstruction. Microsurgery 2010;30:361-7.

13. Angel MF, Im MJ, Chung HK, et al. Effects of combined cold and hyperbaric oxygen storage on free flap survival. Microsurgery 1994;15: 648-51.

14. Shaw WW, Ko CY, Ahn CY, et al. Safe ischemia time in free-flap surgery: a clinical study of contact-surface cooling. J Reconstr Microsurg 1996;12:421-4.

15. Werker PM, Kon M, Green CJ, et al. Cold (0 degree C) ischaemic tolerance of latissimus dorsi free flaps in rats: a macroscopic and morphological study. Microsurgery 1994;15:187-92. 